\title{
Rectenna Design with Space Mapping Optimization
}

\author{
Fang Zhang ${ }^{1,{ }^{*}}$ and Xin Liü, b \\ ${ }^{1}$ Bohai University, Jinzhou 121013, China \\ ${ }^{2}$ Harbin Institutes of Technology, Harbin 150001, China \\ akwsensen@163.com, ’bxinliu.hit@gmail.com
}

Keywords: Computer-aided design (CAD); Engineering optimization; Space mapping; Rectenna

\begin{abstract}
This paper presents a compact planar rectenna with high conversion efficiency design. To reach the conjugate matching between the antenna and the diode, a space-mapping technology was used to accelerate electromagnetic (EM) optimization process. The system design circle time was much reduced. A $2.45 \mathrm{GHz}$ rectenna system was designed, fabricated and measured to guarantee their microwave performance.
\end{abstract}

\section{Introduction}

A rectenna is an RF power receiver that converts the received power into dc power that can then be consumed by other subsystem. In modern wireless power applications, the factors such as cost, size, performance, and design cycle time are needed for design consideration. From the published literatures [1,2], rectenna system design is not simple, which commonly includes antenna, filter, and matching network. From the simulation point of view, most of the design work need system EM simulation to guarantee the performance requirements $[1,3]$, and these cause to increase the design cycle time a lot. In this paper, a folded dipole rectenna is proposed, and a space-mapping technology is used to accelerate EM optimization process, which can solve this issue.

\section{Rectenna Design}

In modern wireless system, besides simple structure, compact size, and low cost, the design cycle time is also an important factor for success of the product. In this section, the particularity of the proposed compact rectenna system is proposed, and a Space-Mapping technology is used to accelerate the final EM optimization process.

Rectenna Design Method Based on Non-Fifty-Ohm Antenna. The rectenna system consists of the passive parts which include antenna, matching network, and filter, and active parts which include diode and other elements of the rectifying circuit. The standard method is to design the antenna, matching network, filter, and rectifying circuit part by part, and combined them for final evaluation. Some coupling effects in this method are neglected at some level. Such method is widely used in the rectenna system design, especially for the $50 \mathrm{Ohm}$ interface rectenna system. To see the real time rectenna performance, a global time domain evaluation method is also reported [3]. However, it also separates the antenna part from the system first, and makes an equivalent circuit from EM simulation. The final system evaluation is done in time domain by the PSPICE. In addition, to measure performances of the antenna and other parts of the system, most of the published system designs are based on $50 \mathrm{Ohm}$ interface, which also limits the rectenna design methods to a narrow range. It is plain to see that the more parts the system uses, the higher the possibility of making the system a larger size and a greater power loss. In other words, theoretically, if the $50 \mathrm{Ohm}$ interface rule is not used, a rectenna still can be considered a complete unit. It also opens the possibility of obtaining a highly integrated rectenna system with a more compact size, low power consumption, and higher conversion efficiency. For convenient explanation, the schematic of the proposed compact rectenna is drawn in Fig. 1. In the proposed rectenna, the diode is directly connected with the antenna. The 
antenna input impedance should be conjugate matching with the diode, which leads to a requirement of non-fifty-Ohm antenna design.

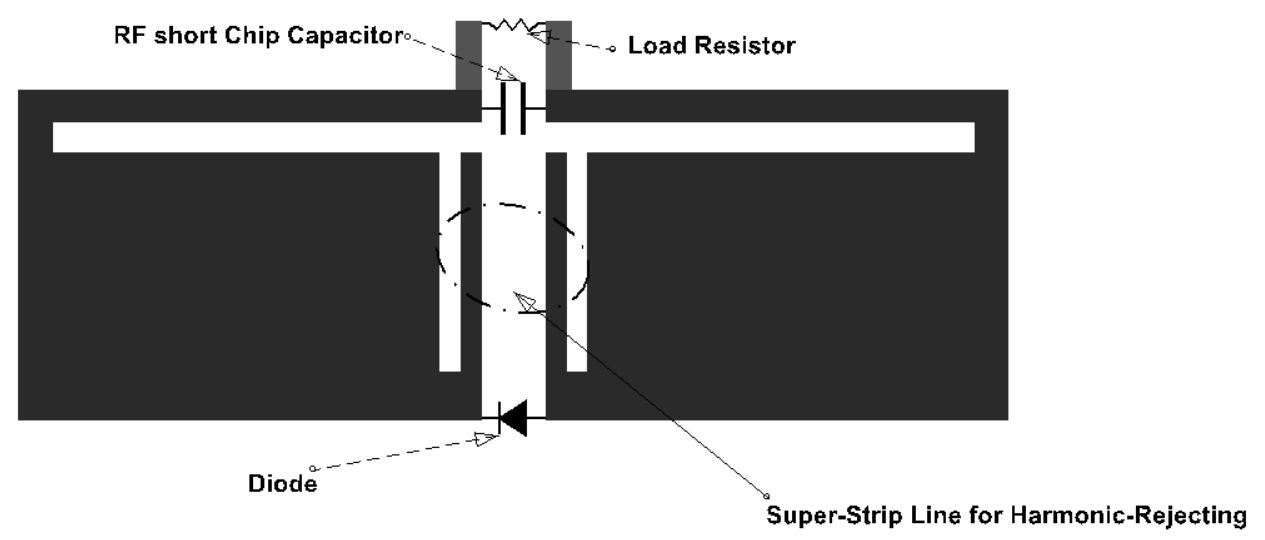

Figure 1. Schematic of proposed compact rectenna

2.45 GHz Rectenna Design. This section addresses the design procedure of a proposed rectenna in the industrial, scientific and medical (ISM) frequency of $2.45 \mathrm{GHz}$. The general design will start from studying the diode. Diode as the key component for rectifying circuit plays an important role for success of the rectenna system. Several characteristics are important in selecting the diode for rectenna system. If higher order harmonic signals are generated from the diode, the system has more possibility with low conversion efficiency. Therefore, a diode with low harmonic generation feature is more suitable in rectenna system. To increase a DC output power from the rectifying process, it is necessary to use a diode of higher break down voltage and larger power consumption. In this design, a HP HSMS-8101 Scotty diode is selected. Considering the power level of the amplifier available in the measurement step, the received power of $8 \mathrm{dBm}$ is chosen for calculating the conjugate matching impedance of the diode. The impedance of the diode is related with both power and the load impedance. At this work, Agilent Advanced Design System (ADS) 2009 is used to analyze the conjugate matching point of the diode at the specified power level. The large signal simulation setup is shown in Fig. 2. When the $8 \mathrm{dBm}$ power adds to the diode, the conjugate matching value can be obtained from optimization in several seconds. The calculated conjugate matching impedance of the diode in Smith chart is shown in Fig. 2 (b) at this design, when the conjugate matching condition is reached, the calculated diode impedance from simulation is $115.461-\mathrm{j} 260.633 \mathrm{Ohm}$ and the port impedance is $115.315+\mathrm{j} 260.899 \mathrm{Ohm}$. The next work is the antenna design with the desired impedance for conjugate matching with the diode. The substrate used is $0.54-\mathrm{mm}$-thick Teflon substrate (dielectric constant $=2.54$ ) with $0.018-\mathrm{mm}$ copper cladding. $100 \mathrm{Ohm}$ load resistor is chosen in this design. The value of the thin film chip capacitor is obtained from the official components library. To get the lower insertion loss at the center frequency of $2.45 \mathrm{GHz}$, GRM18/0603/C0G 5.6 pF capacitor is selected from Murata manufacturing company. To reach the conjugate matching for the final design, the antenna should be studied and optimized in EM simulation. Since optimization using the EM simulation will take much design time, a space mapping technology is used to accelerate the final optimization process.

Space-Mapping Approach to accelerate EM optimization. Space-Mapping technologies focus on reducing unnecessary time-consuming, full-wave EM simulations of microwave structures in modeling and design optimization [4-9]. Space-Mapping suppose the existence and exploitation of "fine" and "coarse" models. The "fine" model may be a CPU-intensive EM simulator that provides high accuracy. The "coarse" model is a simplified demonstration, typically an equivalent circuit or empirical formulas. Implicit space mapping optimization techniques exploit available pre-assigned parameters in the coarse model. The pre-assigned parameters are a set of parameters that are normally 
fixed during the design optimization process, such as dielectric constant and substrate height. Changing the values of some of these parameters, however, is expected to have a similar impact on the responses of the microwave components as does changing the designable parameters. In each iteration, we calibrate these pre-assigned parameters in the coarse model against the fine model (where the pre-assigned parameters are always fixed). A re-optimization is carried out on the calibrated coarse model (surrogate). The new design parameter values are then assigned to the fine model. These steps are repeated until the specifications are satisfied.

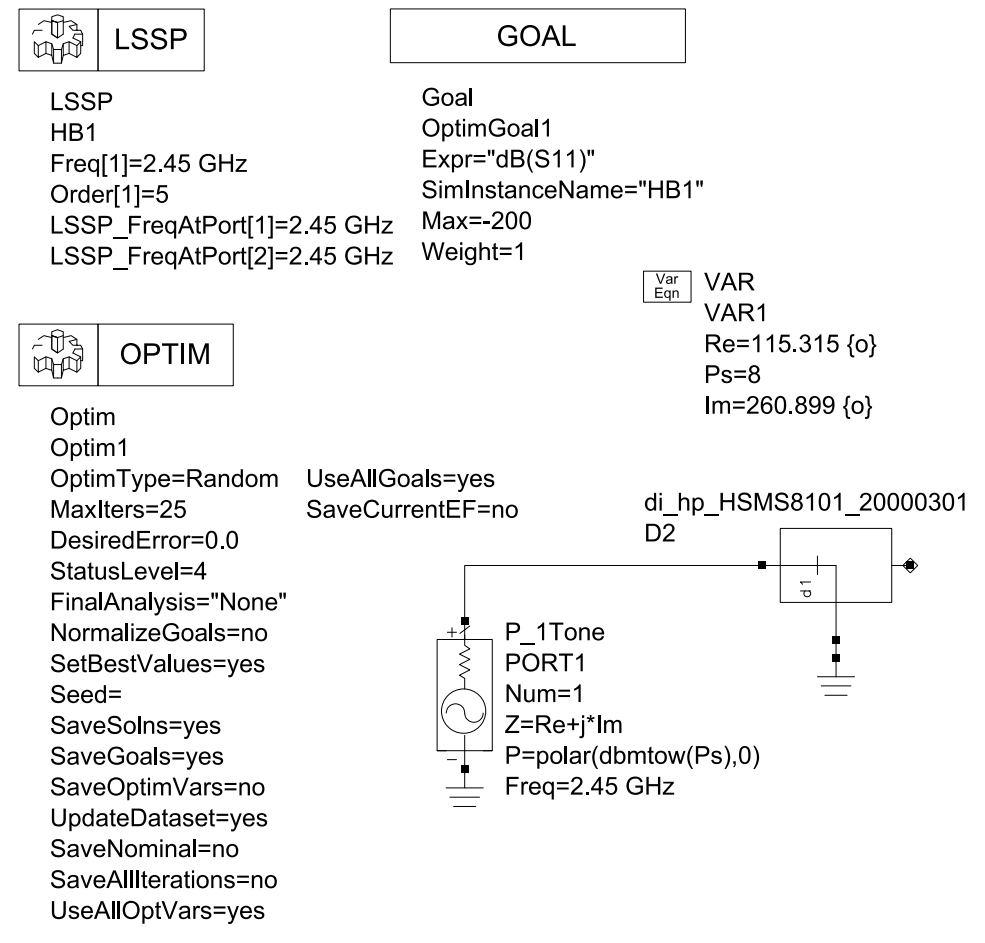

(a)

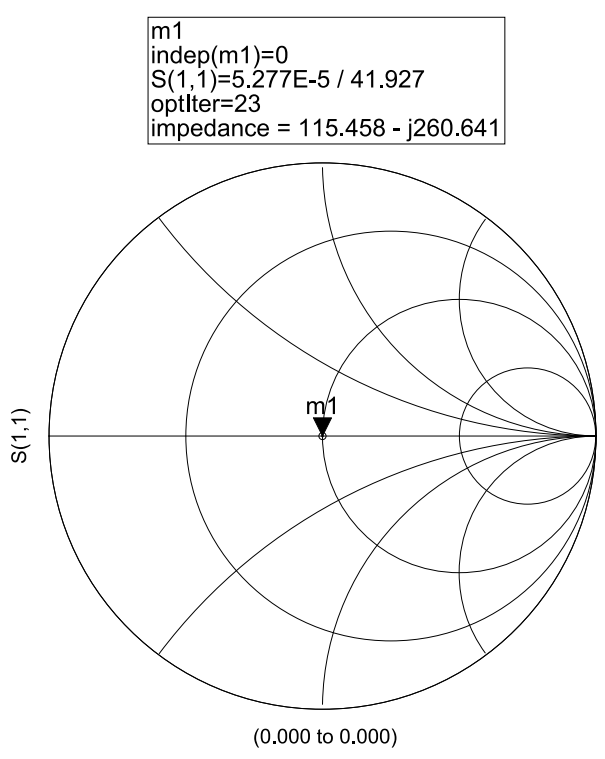

(b)

Figure 2. (a) Setup for calculation of the conjugate matching impedance of the diode and (b) the calculated conjugate matching impedance of the diode in Smith chart

Tuning is a built-in functionality of Agilent ADS [10]. When a coarse model is designed in ADS, its parameters could be set as tunable. In the ADS coarse model, we tune the design and pre-assigned parameters which are the substrate heights and the dielectric constant values in this design. This tuning allows us to note graphically how the parameters affect the responses. With tuning we can break down or split the rectenna parameters of size and examine their effects. In each iteration a better fine model solution is obtained fine models from IE3D Zealand, the tuning-aided space mapping process is implemented in the Agilent ADS system and IE3D. In seven iterations, an accurate design solution is obtained. The implementation takes full advantage of the Agilent ADS tuning technology.

\section{Fabrication and Experimental Study}

The final optimized rectenna is fabricated and measured. The measurement setup and conversion efficiency calculation is followed by [1], except using a $9.8 \mathrm{dBi} 2 \times 2$ patch array replaced by the horn antenna as the transmitting antenna. A DC voltage meter is used for measuring the output DC voltage. The measurement distance is set as $1.2 \mathrm{~m}$. The RF-to-DC conversion efficiency is defined as 


$$
\eta=\frac{\frac{\left(V_{D C}\right)^{2}}{R_{L}}}{P_{\text {received }}}
$$

To calculate the system efficiency, the proposed folded dipole rectenna gain is needed. However, the proposed folded dipole antenna is not designed based on $50 \mathrm{Ohm}$. They are designed based on the complex load impedance, so it is hard to directly measure the proposed antenna gain accurately in that situation. To calculate the conversion efficiency, a $50 \mathrm{Ohm}$ wideband folded dipole antenna operation in the same center frequency of the rectenna is designed and fabricated to measure the received power. Then, the received power of the rectenna is calculated from the measured received power. It should be pointed out that in conventional method of calculation, the rectenna conversion efficiency, the gain of the antenna with filter network is needed for design and measurement without considering the load and diode. Since the proposed rectenna gain is simulated including all the system components, it is a more systematic design method than the conventional calculation method. It is also very suitable for the first-pass system success. Finally, the output voltage is measured and the conversion efficiency is calculated and plotted in Fig. 3.
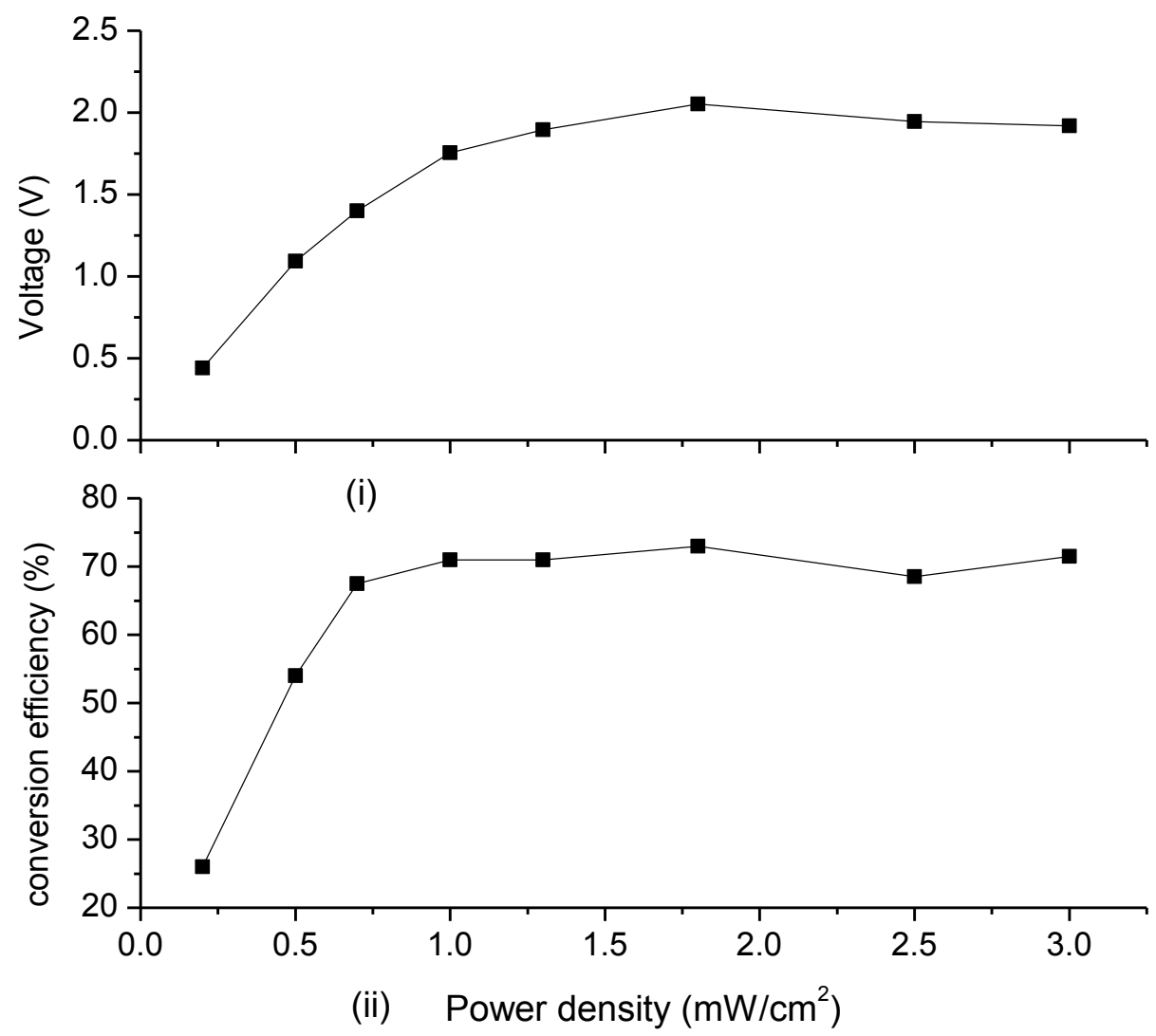

Figure 3. Measured DC output voltages and Calculated conversion efficiencies

\section{Conclusion}

A compact architecture rectenna system has been developed. It has been shown that the proposed rectenna system simplified the design work. The proposed rectenna has a compact size, unit-planar structure, and low cost. To reduce the design time in the final EM simulation, a space mapping technology is evolved. With tuning-aided implicit space mapping, the speed and accuracy of the final optimization was much enhanced. Considering the above advantages, the proposed rectenna with space mapping optimization method is very suitable for the for the first-pass system success. 


\section{Acknowledgements}

This work is supported by National Nature Science Foundation of China under Grant No. 61401044 and No. 61401117.

\section{References}

[1] Y. Suh and K. Chang: IEEE Trans. MI crow. Theory Tech., Vol.50, (2002) No.7, p.1784-1789.

[2] T. Yo, C. Lee, and C. Luo: MI crow. Opt. Technol. Let. Vol.50, (2008) No.1, p.45-48.

[3] B. Esaki, G. Akoun, and L. Pichon: Int. J. Numer. Model. Vol.20, (2007) No.1, p.3-15.

[4] J.W. Bandoleer, Q.S. Cheng, S.A. Dakroury, A.S. Mohamed, M.H. Bakr, K. Madsen, and J. Sønder- gaard: IEEE Trans. MI crow. Theory Tech., Vol.52, (2004) No.7, p.337-361.

[5] S. Yin, X. Li, H. Gao, O. Kaynak: IEEE Trans. on Ind. Electron., Vol.62, (2015) No.1, p.657-667,.

[6] S. Yin, Z. Huang: IEEE/ASME Trans. on Mecha.Vol.20, (2015), No.5, p.2613-2620.

[7] S. Yin, X. Zhu, O. Kaynak: IEEE Trans. on Ind. Electron., Vol.62, (2015), No.3, p.1651-1658.

[8] S. Yin, X. Zhu: IEEE Trans. on Ind. Electron., Vol.62, (2015), No.6, p.3852-3861.

[9] S. Yin, O. Kaynak: Proceedings of the IEEE, Vol.102, (2015), No.3, p.143-146.

[10] Agilent Advanced Design System, Ver. 2005A, Agilent Technologies, Santa Rosa, CA, 2005. 